my journal manuscript No.

(will be inserted by the editor)

\title{
Solar Sector Structure
}

\author{
Hugh Hudson • Leif Svalgaard · Iain \\ Hannah
}

Received: date / Accepted: date

\begin{abstract}
The interplanetary magnetic field near has a characteristic "sector" structure that reflects its polarity relative to the solar direction. Typically we observe large-scale coherence in these directions, with two or four "away" or "towards" sectors per solar rotation, from any platform in deep space and near the ecliptic plane. In a simple picture, this morphology simply reflects the idea that the sources of the interplanetary field lie mainly in or near the Sun, and that the solar-wind flow enforces a radial component in this field. Although defined strictly via the interplanetary field near one AU, recent evidence confirms that this pattern also appears clearly at the level of the photosphere, with signatures including not only the large-scale structures (e.g., the streamers) but also highly concentrated fields such as those found in sunspots and even solar flares. This association with small-scale fields strengthens at the Hale sector boundary, defining the Hale boundary as the one for which the polarity switch matches that of the leading-to-following polarity alternation in the sunspots of a given hemisphere.
\end{abstract}

Keywords First keyword · Second keyword · More

\author{
H. S. Hudson \\ Space Sciences Laboratory \\ University of California \\ Berkeley CA 94720 USA Tel.: +1-510-643-0333 \\ E-mail: hhudson@ssl.berkeley.edu \\ L. Svalgaard \\ W.W. Hansen Experimental Physics Laboratory, Stanford University, Palo Alto CA USA \\ I. G. Hannah \\ SUPA School of Physics \& Astronomy University of Glasgow Glasgow, UK
}




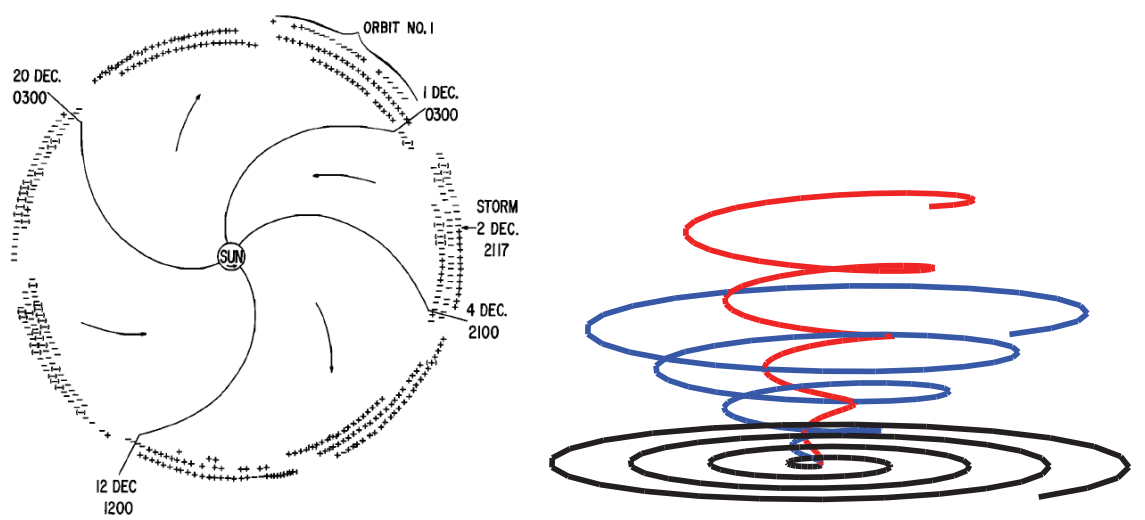

Fig. 1 Left, an early view of the interplanetary magnetic sector structure, from Wilcox and Ness (1965). The symbols reflect the predominant orientation of the interplanetary field in 3-hour data segments from the magnetometer on board the IMP-1 spacecraft, anchored at Earth but with an apogee $\left(31.7 R_{E}\right.$, orbital period about $\left.3.9 \mathrm{~d}\right)$ large enough to give clear views of the solar wind in the ecliptic plane. Plus and minus conventionally represent outward and inward field directions; this early sample shows a persistent four-sector pattern. Right, a sketch of how field lines at 0,30 , and $60^{\circ}$ heliolatitude must look in an isotropic high-beta solar wind with constant radial speed (from Owens and Forsvth 2013).

\section{Introduction}

The early years of human exploration of space beyond the Earth system saw the development of instrumentation capable of detecting the then-hypothetical solar wind, and then the magnetic field embedded within it. Qualitative physical arguments had made it clear that the field stretches out in the radial direction, and that it eventually must adopt a spiral pattern in the ecliptic plane (Biermann 1957; Alfvén 1957; Parker 1958). The mean speed of the observed solar wind dictates that this spiral should have angle of about $45^{\circ}$ to the radial at one $\mathrm{AU}$, and that the essentially radial flow should take 4-5 days in transit. Observations generally confirmed these rough ideas.

The first interplanetary space probes capable of sufficiently sensitive magnetic measurements revealed the existence of the sector structure (Wilcox and Ness 1965). Figure 1 (left) shows a data representation from that era, which illustrates the basic idea. This remarkable feature of the solar magnetic field, as evidenced deep in the heliosphere but at heliolatitudes near the ecliptic plane, immediately demanded an understanding of the relationship between the different domains that the "towards" and "away" sectors represented. The nature of the underlying solar magnetic field as revealed by magnetographic observations of the photosphere became a hot topic. The 3D nature of the heliosphere (Figure 1 right ) compounded the complexity of these questions, since the geometry of a sector could not be readily inferred from near-ecliptic observations.

In a major development, Svalgaard (1972) established that one can use polar geomagnetic records to determine the times when Earth passed through 
a sector boundary. This opened the possibility of a proxy record of the global development of the heliospheric field that in principle can extend into the middle of the 19th century. We discusss this in more detail in Section 3 here. Shortly thereafter Gulbrandsen (1973) and Antonucci and Svalgaard (1974) established a correlatopm between the sectpr pattern at one AU with coronal green-line (Fe XIV) structures (Section 4) in the middle corona. This finding directly related the heliospheric sector structure to small-scale solar magnetic fields.

In this review we describe (Section 5) the recent further identification of the solar footprints of the sector patterns to the level of the photosphere (Svalgaard et al. 2011; cf. Svalgaard \& Scherrer 2014). This involves the concept of the "Hale sector boundary," defined by Svalgaard and Wilcox (1976) as that part of the sector boundary at which the polarity change matches that of the change of polarity from preceding to following sunspot (Section 5).

In retrospect, it may seem reasonable that a heliospheric magnetic domain structure must exist, and that the domains must simplify into sector patterns at great distances from the solar surface. The Sun possesses strong magnetic fields that originate in current systems largely contained below the photosphere; we know this because of the fairly good typical match with simple potential-field expansions in the lower corona (Section 4). This means that if one makes a multipole harmonic extrapolation of the very complicated pattern of photospheric magnetic fields - which evolves with large-scale organization, but also in seemingly random ways - one predicts that the lowest-order term, the dipole, will eventually dominate the field and become the only surviving component at large radial distances. This reasonable viewpoint ignores the fact that the field finds itself embedded in an active plasma, through which large-scale currents flow even far from the Sun. In fact, the heliospheric magnetic field - as opposed to the coronal field - has a maximally non-potential structure according to the Aly-Sturrock theorem (Alv 1991; Sturrock 1991). It contains the large-scale current sheet needed to reverse the field direction between hemispheres.

\section{Solar magnetography and the sector structure}

Hale's discovery of Zeeman splitting in solar spectra has led to extensive, but tantalizingly incomplete, knowledge of the patterns of solar magnetism in the photosphere, and now to major efforts in the observational characterization of the magnetic field in the upper solar atmosphere; we return to this in Section 4. In the era of discovery of the interplanetary sector structure, our remotesensing knowledge of the solar magnetic field mainly consisted of synoptic maps, at low resolution, of the line-of-sight component. Measurement of the full vector field has now become routine, but problems of interpretation remain; Borrero and Ichimoto (2011) discuss many of them in a recent review. We return to this topic in Section 4 , and continue roughly historically here. 
One essential problem is that resolution has improved to the point where the atmospheric dynamics and the characterization of the magnetic field require a simultaneous solution via numerical simulations. The numerical approach will eventually help to solve the fundamental uncertainty in the physical heights of atmospheric structures. The standard semi-empirical modeling of the solar atmosphere (e.g., the VAL-C model of Vernazza et al. 1981) scales with optical depth, rather than height directly, and has an upper boundary at $2543 \mathrm{~km}$ above the photosphere as normally defined in terms of optical depth unity at $5000 \AA$. Such models make many strong physical assumptions, for example in imagining a static structure that can be described as onedimensional. The density structure of the corona is best known only from the ill-posed 3D reconstructions of its projected brightness from projected data in one or two planes (e.g., Aschwanden et al. 2008b, a), or via rotational synthesis that has inevitable confusion with K-coronal time variations (Frazin et al. 2010). Any quantitative understanding of field gradients must of course have precise geometrical information. Nevertheless even the line-of-sight Zeeman measurements provide a wealth of morphological detail across the entire face of the Sun; we understand a great deal even at this level of observational technique.

Typical line-of-sight solar magnetograms turned out to give no clues at all to the physical origin of the domains that the sectors represent, and early representations searched in vain for simple rigidly-rotating zonal patterns in the photosphere - rigidly rotating, because one can derive a precise angular velocity from studying the phase of sector-boundary crossings. Figure 2 shows the stability of the sector pattern over a period of many years. It represents the deviation of sector-boundary arrivals relative to a fiducial 27-day synodic rotation rate (Svalgaard and Wilcox 1975). The stability of these phase measurements suggests a source that has only a slowly-varying rotation law.

Some of the mystery regarding the solar origins had been resolved by the discovery of the heliolatitude dependence of the sector patterns (the RosenbergColeman effect), clearly recognizable in the early data from outside the ecliptic plane (Rosenberg and Coleman 1969). This led to the concept of a single warped current sheet (e.g. Schulz 1973; Svalgaard et al. 1975) extending to large radial distances; its intersections with the ecliptic plane defines the sector boundaries seen there, and at sufficiently high heliolatitudes it must have a unipolar character; this concept extended the model proposed by Svalgaard et al. (1974) and attained great prominence as the heliospheric "ballerina skirt." The Ulysses mission triumphantly confirmed this picture (e.g., Balogh and Erdös 2013), consistent with other data such as the radio (interplanetary scintillations) finding of high-speed solar wind streams at high heliographic latitudes (Coles and Rickett 1976) and the X-ray finding of polar coronal holes (Vaiana et al. 1973).

The presence of an initially mysterious sector structure in the interplanetary field (e.g., Wilcox and Howard 1968) led to the development of synoptic observing programs (see also Section 5). Wilcox and his collaborators at Stanford University created what is now called the Wilcox Solar Observatory, which 


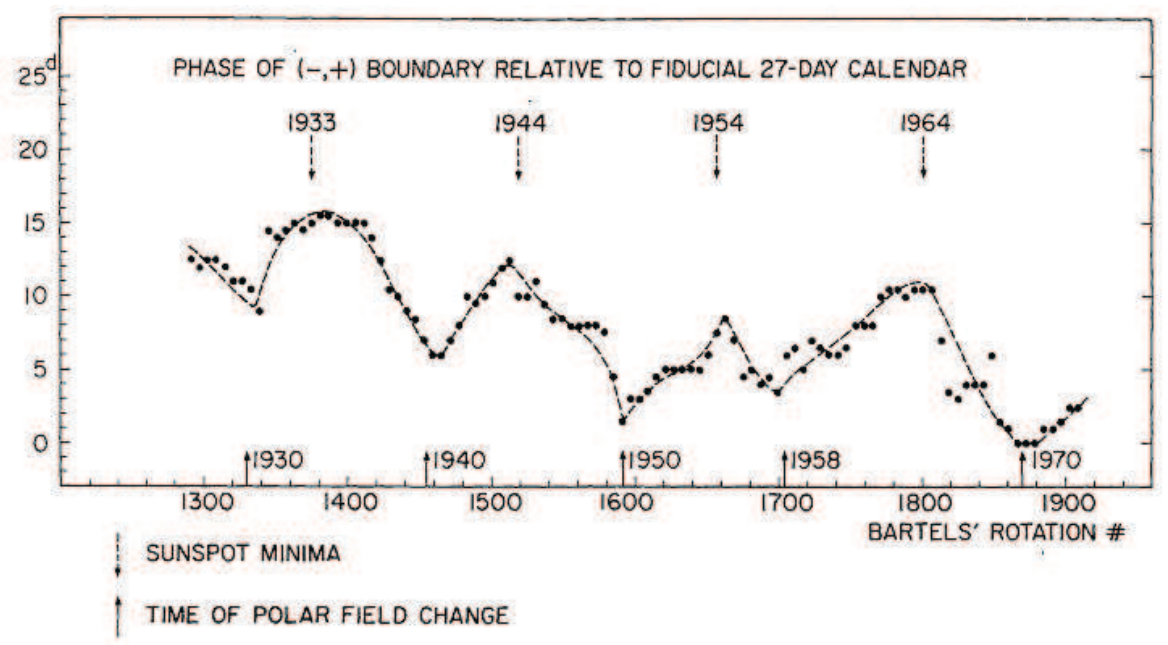

Fig. 2 Phase of sector-boundary crossing, relative to a fiducial 27-day rotation law (Svalgaard and Wilcox 1975).

has provided a long-running data set of low resolution but great stability. This facility began collecting data in 1975 and continues to the present time.

\section{Geomagnetism and the sector structure}

The interplanetary magnetic field in the vicinity of the Earth interacts with the Earth's own field in a complicated manner; the orientation and strength of the field in the incoming solar wind perturb the geomagnetic field as measured on the surface of the Earth. Given a terrestrial field of order $1 \mathrm{G}$ and an interplanetary field of a only a few $\times 10^{-5} \mathrm{G}$, one might expect little effect, but in fact the signatures could readily be measured even with the techniques available in the 19th century (the Gauss-Weber variometer). The sector structure thus has a proxy record that extends quite far back in time. Svalgaard (1968) and Mansurov (1970) showed that the interplanetary magnetic field produced a characteristic pattern of diurnal variation at high-latitude sites such as Thule $\left(12.5^{\circ}\right.$ geographic colatitude) or Vostok $\left(168.5^{\circ}\right)$. The Thule station has a geomagnetic colatitude of only about $5^{\circ}$, and Svalgaard (1973) notes that the major effects of this perturbing current system lie with $15^{\circ}$ of the geomagnetic pole; he attributes the characteristic variation to a Hall current (Baker and Martyn 1953) typically extending about that distance from the pole, and circulating with opposite senses for away and towards sectors. The morphology of this diurnal effect distinguishes it sharply from others such as those of auroral electrojets.

This Svalgaard-Mansurov effect (Wilcox 1972) is strong enough to produce reliable determinations of the polarity of the interplanetary field (Friis-Christensen et al. 1971), usually even on a daily basis (Vokhmyanin and Ponvavin 2013), and so 


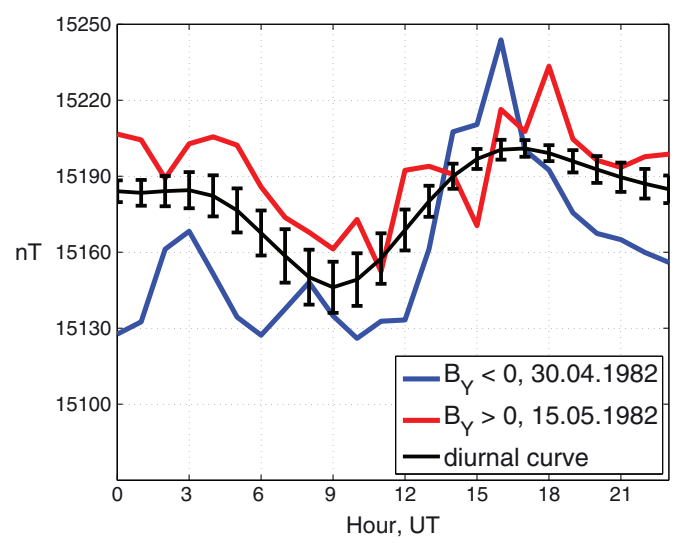

Fig. 3 Illustration of the Svalgaard-Mansurov effect using modern (1982) data from the Nurmijärvi station, which continued the 19th-century Helsinki observations. The black line is the mean diurnal variations, and the red and blue are data from days of known away (red) and towards (blue) sectors, showing the clear distinction between the two orientations.

it serves to extend the record of Earth's sector-boundary crossing times back into the 19th century. This relies upon the understanding of the available geomagnetic observations at sufficiently high latitudes. Vokhmyanin and Ponyavin (2013) published sector-boundary data for Carrington cycles 9-13 (1843-1902) in this way, for example, by making use of the records from the now-discontinued Helsinki $\left(29.8^{\circ}\right.$ geographic colatitude) and St. Petersburg $\left(30.0^{\circ}\right)$ geomagnetic observatories. Figure 3 illustrates this heliospheric effect on the the Earth's polar field, calibrating the residuals away from the mean diurnal variation of Nurmijärvi data by reference to known sector orientations. This observatory produces results closely matching those of the original Helsinki observatory (Nevanlinna 1997).

\section{The corona, the solar wind, and the sector structure}

In this section we discuss three perspectives on the sector structure: from the heliosphere, from the corona, and from the photosphere. The information from these domains have different qualities (in situ or remote-sensing), and are never complete, but generally agree now upon most of the basic structure. We discuss one exception to this understanding in Section 5.

\subsection{As viewed in the heliosphere}

\subsubsection{Observations in situ}

The identification of solar sources of the interplanetary sector structure began with Gulbrandsen (1973) and Antonucci and Svalgaard (1974), who found a 
remarkable long-term agreement between the sector boundaries, appropriately time-shifted, and coronal photometry in the "green line" of Fe XIV (5303 $\AA$ ). We return to this important result in the next section, but first briefly describe the nature of the corona as seen in the green line or in various other ways.

One description of the solar corona envisions it as a concentric spherical shell of relatively low plasma beta (i.e., in force balance mainly via the Maxwell stress tensor); on intermediate time scales it is indeed quite stable but is prone to sudden and drastic disruptions, often on large scales, related to the domain structure of its magnetic connectivity. The lower boundary - subject to any of several possible definitions - has corrugations imposed by the dynamics of the lower solar atmosphere, and the upper boundary fades into the solar wind in a manner that probably defies observation; both the upper and lower boundaries thus presumably have distinctly non-spherical shapes.

This complicated-sounding region has a simple and attractive model that describes its geometry fairly well: the "potential-field source-surface" (PFSS) model of Schatten et al. (1969) and Altschuler and Newkirk (1969). Such models typically have a driver at the lower boundary from the line-of-sight Zeeman magnetic measurements, interpreted globally via a synoptic map of the measurements at central meridian. Given the foreshortening toward the polar regions, this approach clearly omits a great deal of spatial and temporal structure that the EUV or X-ray movies, for example, reveal on many scales. Based on this boundary, the PFSS model consists of a potential-field extrapolation out to an ad hoc "source surface," typically a spherical boundary at which a fictitious current system forces the exterior field into a strictly radial configuration. This radial field then constitutes the "open" magnetic flux that somewhat mysteriously 1 fills the heliosphere via advection in the solar wind. See for example Lockwood (2013) for a discussion of subtle points in the estimation of heliospheric open field and its significance. A common choice for the radius of the source surface, $2.5 \mathrm{R}_{\odot}$ (Hoeksema et al. 1983), yields an approximate match to the heliospheric flux, which appears to vary by a small factor across the solar cycle.

We note that the heliospheric data make it possible to measure the open flux directly, in the sense of identifying it with the radial component $\mathrm{B}_{r}$ of the field. The Ulysses data showed, remarkably, that there was a minimal latitude dependence of this quantity (Smith and Balogh 1995), so with that fact and the inverse square law, one can derive a good estimate of the open flux from any point in the heliosphere. Again remarkably, given the weaknesses inherent in the photospheric data, the direct view in terms of $\mathrm{B}_{r}$ turns out to agree reasonably well with that derived from PFSS with a fixed source surface (Owens et al. 2008; Wang and Sheeley 2003). Figure 4 sketches the long-term variations of solar open flux as determined by these methods.

The Ulysses "fast latitude scans" also provided an excellent overview of the $3 \mathrm{D}$ sector structure as it related to the solar wind, as illustrated in Figure 5 , This view nicely suggests how the basic pictures in Figure 1 work themselves

1 How can a radial flow advect magnetic field parallel to itself? 

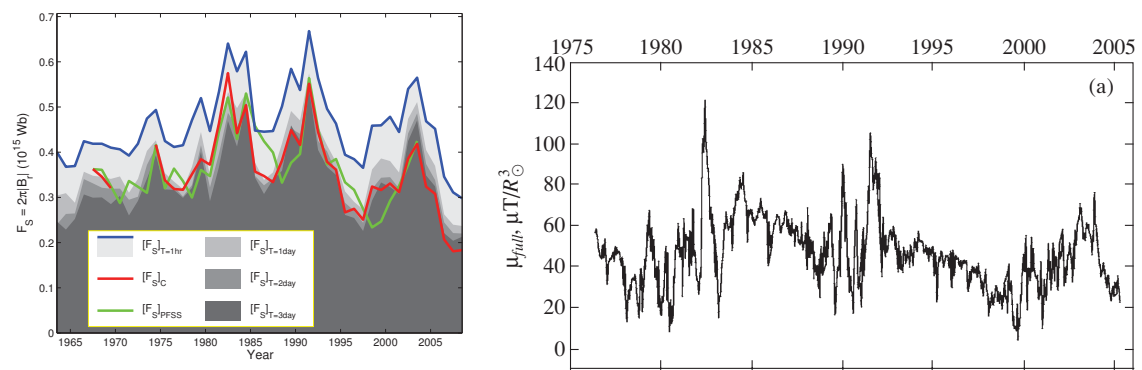

Fig. 4 Left, the solar open flux estimated via the PFSS method (red line) and heliospheric sampling (from Lockwood et al. 2009); right, estimates of the amplitude of the best-fit dipole term for the photospheric field (Livshits \& Obridko, 2006).

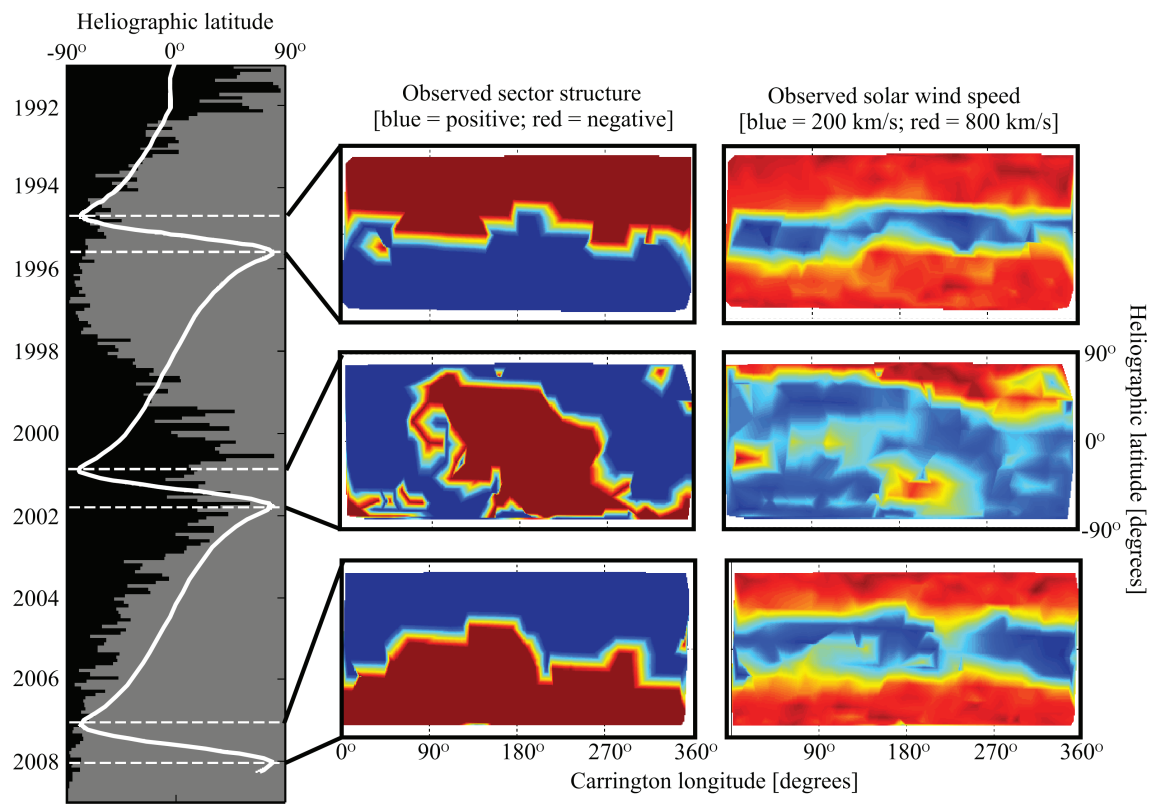

Fig. 5 The three rapid scans of heliolatitude executed by the Ulysses spacecraft: left, the heliolatitude and sunspot number; middle and right, source-surface projections of polarity and wind speed, respectively (from Owens and Forsvth 2013). The Ulysses orbital inclination of $80.2^{\circ}$ and perihelion of $1.3 \mathrm{AU}$ meant that each full scan required about one year.

out in three dimensions, at least with this crude sampling; each map corresponds to the entire year of the "fast" scan and thus presupposes great long-term stability (Owens et al. 2011). Note that the small regions of minority polarity in the middle panels may just reveal artifacts resulting from this sampling; see Antiochos et al. (2012) for a theoretical discussion of the heliospheric magnetic connectivity. 


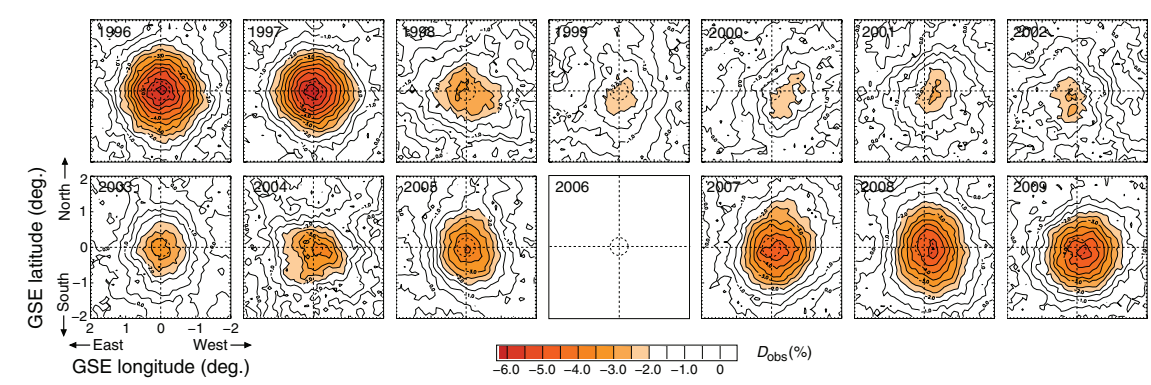

Fig. 6 The time development of the observed cosmic-ray shadow of the Sun, with its coronal magnetic field, from the Tibet air-shower array (Amenomori et al. 2013). Year 2006 had insufficient statistics and the figure omits its image.

\subsubsection{Remote-sensing observations}

The idea of using the cosmic-ray shadows of large structures (Clark 1957) has developed into a method for probing the coronal magnetic field (Amenomori et al. 1993; Amenomori et al. 2013). This observation uses TeV-energy primary cosmic rays detected via their extensive air showers, and the first results immediately showed a significant modification of the solar cosmic-ray shadow depending on the presence of towards and away sectors. Since this 1993 paper, the data have accumulated and improved to the point where more sophisticated analyses for coronal magnetic-field structure have become possible. Figure 6. from Amenomori et al. (2013), shows the development of the cosmic-ray shadow detection over a solar-cycle time span.

A quantitative understanding of the solar magnetic shadow involves complex forward-method simulation. Amenomori et al. estimate the FHWM angular resolution and modal energy of the Tibet air-shower data as $0.9^{\circ}$ and $10 \mathrm{TeV}$, respectively. Because of the small event rate, and the shallowness of the shadow, long integrations are required to obtain significant results. These results depend upon Monte Carlo simulations of cosmic-ray transport and interaction within the inner heliosphere, for which a time-resolved magnetic model (e.g., the PFSS) is necessary. The observations shown in Figure 6 permitted the authors to distinguish a standard PFSS model from a more elaborate current-sheet model Zhao and Hoeksema (1995). More elaborate data analyses may allow us to follow the structure of the heliospheric field in the relatively unknown domain at the distance of the standard source surface, $2.5 \mathrm{R}_{\odot}$, and of course it would be most interesting to be able to characterize the development of the warp structure of the heliospheric current sheet in this region. 
4.2 As viewed in the corona

Understanding the magnetic field in the solar corona directly requires remotesensing astronomical measurements (e.g., for Zeeman splitting), which can be exceedingly difficult. One can also interpret the images to obtain geometrical information from the orientations of the striations (e.g., Aschwanden 2013). The image-interpretation approach has improved continuously with the development of higher spatial resolution and the better sampling afforded by modern observations, because the images observable in the emission corona have striations at the finest observable scales. These striations arguably guide us to the orientation of the field threading the plasma. The gyroresonance condition also allows magnetography via microwave emission, especially in active regions; for example, Brosius and White (2006) found an example of $\mathrm{kG}$ fields at $\mathrm{Mm}$ heights via this technique. None of these approaches though provides detailed direct information about $\mathbf{B}$ everywhere in the corona.

Discerning the full geometry of the coronal field thus requires modeling. The corona presents a serious geometrical problem here; for the most part it is optically thin, and so the line-of-sight depth cannot be known observationally. The PFSS approach described in the previous section is the most frequently used method, but there are many other approaches with various degrees of sophistication (e.g., De Rosa et al. 2009; Mackay and Yeates 2012), mostly involving the assumption of a force-free field. Beyond the purely mathematical treatments, which might suffice for an idealized corona at low plasma beta and no chromosphere, there are various efforts to incorporate plasma physics in various approximations, as also reviewed by Mackay \& Yeates.

The concept of the "web of separatrices" or S-web Antiochos et al. 2007, 2011, 2012) may simplify our view of how the complicated magnetic field of the lower solar corona maps into the relatively simple one in the heliosphere; in the ideal case of no sunspot activity whatsoever, this heliospheric field tends to become radial while retaining its bipolar character. Figure 7 shows the Sweb for a snapshot synoptic diagram at solar minimum. Within the S-web structure one envisions magnetic reconnection to occur in such a manner that closed-field plasma, enriched in high-FIP elements, can escape into the slow solar wind over the its full breadth in heliographic latitude (e.g., Zurbuchen 2007). A diffusive process with similar consequences underlies the "interchange reconnection" theories (cf. Fisk and Schwadron 2001; Fisk and Zhao 2009), but the details of both pictures remain somewhat unclear owing perhaps to the difficulty of modeling the local physics of magnetic reconnection within the MHD framework.

The synoptic chart in Figure 7 shows the interesting structure of the Sweb: sometimes it appears to form mainly on one side of the heliospheric current sheet. This azimuthal dependence could be related to the large-scale organization of the field embodied in the Hale boundary (Section 5), but we are unaware of any work describing this possible association or its significance. 

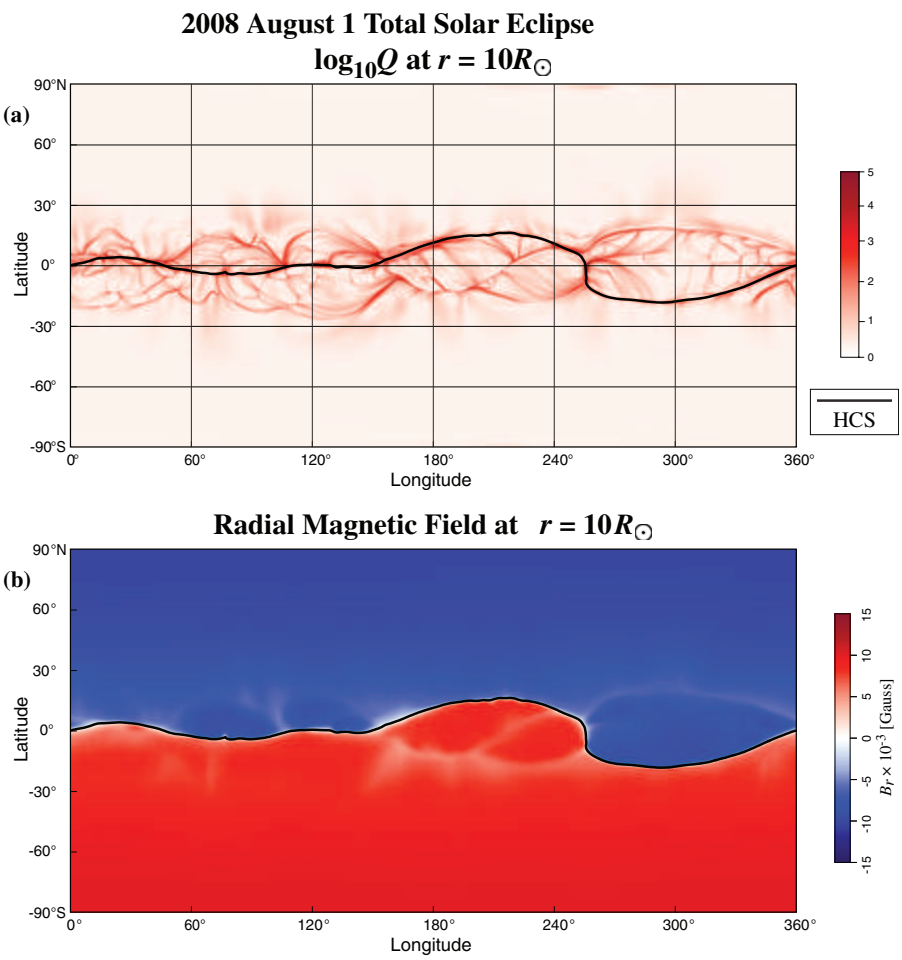

Fig. 7 Synoptic charts illustrating the "Web of separatrices" (Antiochos et al. 2011) for a snapshot at solar minimum. The upper panel shows the "squashing factor" $Q$, essentially the Jacobian of the mapping, with high values representing quasi-separatrix layers (Priest and Démoulin 1995). The lower panel shows the radial field component $\mathrm{B}_{r}$, and on both panels the black line shows the heliospheric current sheet.

\subsection{As viewed at the photosphere}

A substantial development of solar magnetic-field models has proceeded entirely from their photospheric (surface) manifestations, the so-called fluxtransport models following Leighton (1964, 1969). Leighton's "magnetokinematic model" contains a few free parameters, including descriptions of a diffusive motion of vertical magnetic flux at the photosphere, and a meridional circulation there, and successfully describes the main properties of the solar cycle on this basis. These models take their inspiration from the Babcock (1961) picture of how dynamo action may create the solar cycle deep in the interior of the Sun. Curiously, the Leighton flux-transport models succeed quite will in describing the surface manifestations without reference to the interior field structure at all. The coronal field can be modeled in this way (e.g. Wang and Sheeley 1991, 2003), and thence used to predict the properties of the heliospheric fields (Hoeksema et al. 1983), including the locations of the sector boundaries. These predictions do not require us to specify the 
interior volume threaded by the currents that give rise to the field outside the photosphere: deep-seated, as Babcock envisioned, or superficial, as modeled.

The interior-to-corona continuity of the large-scale solar field obviously has a descriptive representation in terms of spherical harmonics, and the behavior of the harmonic terms might give a guide to the physics involved. As noted for example by Wang et al. (2000), the lowest-order terms of the multipole expansion have the strongest effect at the source surface of a PFSS mode because it is at the outer boundary of the domain. By construction, these terms determine the regions of open flux. The variations of the axisymmetric and equatorial dipole terms therefore must dominate the open flux, as noted by many, if the main source of the interplanetary field lies within the body of the Sun. Note the important caveat that we do not actually know how deep these sources may be (or what creates them), as opposed to the case of the Earth with its well-defined core.

\section{Photospheric identification of the sector boundaries}

The early observations of sector boundary crossings showed them to have stable phases relative to fixed periods (Figure 1). This suggested a rigid rotation pattern, rather than a differential one, and yet no solar surface feature has such a property. Subsequently the three-dimensional nature of the sector domains in the heliosphere became more apparent, as discussed above and illustrated in Figure 5. At this point one could have inferred that the domain structure had a relatively simple interpretation: the waxing and waning of the polar coronal holes, and their pattern of polarity reversal, plus the simplification of the harmonic structure imposed by the expansion of the solar wind, could lead to a simplistic view of the structure formation. The theoretical ideas discussed in Section 4 confuse the issue by invoking differing views of the microphysics and its site.

Recent work has opened new issues regarding the solar origins of the magnetic domains of the interplanetary sectors, extending the association first noted by Gulbrandsen (1973) via the green-line corona and its correlation with the sector boundaries. The new work goes right to the level photosphere and identifies sector boundaries directly with small-scale features there, as we describe below. The key to these new associations is the concept of the Hale sector boundary (Svalgaard and Wilcox 1976), as illustrated in Figure 8 . The recognition that the Hale sector boundary systematically correlated with signatures of solar activity could not have been anticipated, and still has no clear interpretation. Geometrically, the sector structure itself seems fixed with respect to the solar rotation (see Figure 1); the various aspects of solar activity also do, though with latitude dependence reflecting the photospheric differential rotation. So, what singles out the Hale boundary portion as a locus of solar activity?

Further and more specific evidence for the linkage of the Hale sector boundary with solar activity came with the discovery that solar flares preferentially 

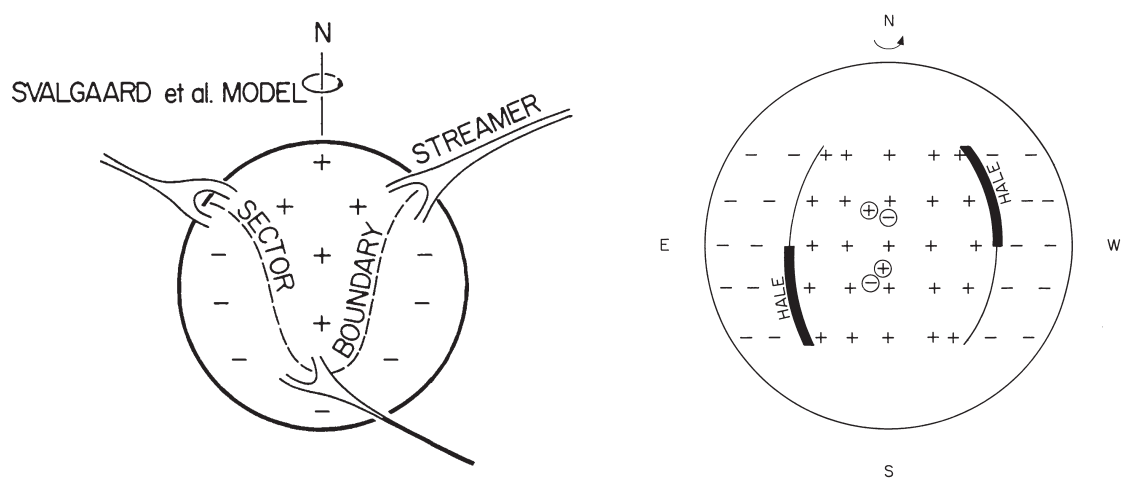

Fig. 8 Left, the now-accepted view of the coronal origin of the interplanetary sectors, as proposed originally by Svalgaard et al. (1974). Right, the "Hale boundary" (Antonucci and Svalgaard 1974), defined as that part of the sector boundary at which the polarity switch matches that of the leading sunspot polarity in the corresponding hemisphere.

occur at the Hale boundary (Svalgaard et al. 2011), a result making use of the RHESSI flare catalog of flare locations (J. McTiernan, private communication 2014). This analysis reflects only the first few years of RHESSI observations, which began in 2002 and thus come from Cycle 23, but a further analysis (Iain Hannah, private communication 2013) extends this to Cycle 24 flares. As noted by Svalgaard et al. (2011), RHESSI is not central here, and the ordinary NOAA flare listings also show the same effect but with reduced spatial resolution.
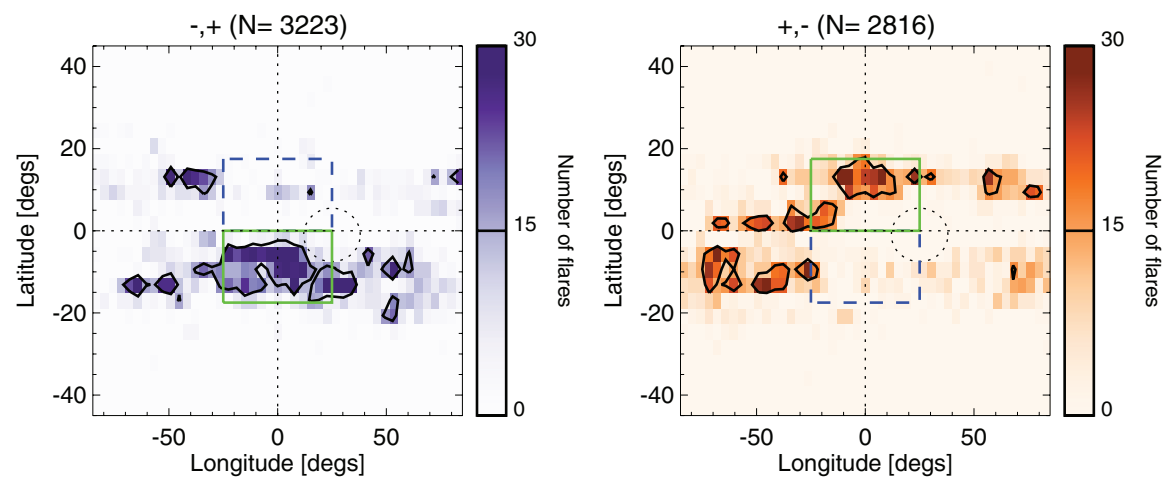

Fig. 9 RHESSI's view of the Hale boundary, for flare occurrence 2002-2010. The colored contours show the locations of flares relative to the times of sector boundary crossings at one $\mathrm{AU}$; the boxes with solid lines show the locus expected for the Hale boundary segment, and the dashed the disfavored. The left and right panels show the $(+,-)$ and $(-,+)$ crossings, respectively (adapted from Svalgaard et al. 2011). 


\section{Long-term evolution of the sector structure}

The long-term behavior of the solar magnetic field, extending many Hale cycles into the past, has theoretical as well as practical interest (the latter since some prediction techniques involve solar activity with patterns whose "memory" extends to the preceding cycle or even earlier). Regularities and irregularities exist on these longer scales, for example in the NS asymmetry discussed by Bell (1962). This paper also made the link between sunspot asymmetry and an even stronger asymmetry in the occurrence of great magnetic storms over the solar cycles between 1833 and 1960. A corresponding asymmetry appears in the Ulysses cosmic-ray data (Simpson et al. 1996), and Mursula and Hiltula (2003) showed this cone-like distortion to be a persistent pattern of behavior. Wang and Robbrecht (2011) then described this in terms of the additional magnetic pressure created in the low corona by the asymmetric eruption of field.

To the patterns of such long-term variations of sunspot, geomagnetic, and heliospheric variation we can now add the morphology of the Hale sector boundary, since it too can be traced well into the 19th century now. Figure 10 shows the result of extending the historical record back to Cycle 9, in the form of summed-epoch analyses similar to those shown in Figure 9. maps of occurrence summed on the key times given by the sector-boundary crossings identified geomagnetically, each showing the distribution as a function of central meridian distance and heliographic latitude. The Figure shows four panels for each of the Odd cycles $(16,18, \ldots, 24)$ and Even cycles $(17,19, \ldots$, 23 ), distinguishing the odd/even crossings from the even/odd crossings and in each case identifying the hemisphere of the Hale boundary. This confirms the association of sector boundaries with flux concentrations and the coincidence of Hale boundaries with the correct hemisphere in each case, and shows the result to be stable over multiple Hale cycles.

\section{What are the solar sources of the heliospheric field?}

An equivalent question might read "Why do sector boundaries correlate with solar activity?" The problem is that the small-scale closed fields of solar active regions, which lead through time to the structure of the streamer belt and the green-line observations (e.g., Antonucci and Svalgaard 1974), must appear as dome-shaped inclusions of more intense field in the low corona. Conceptually, the dome becomes larger as more flux emerges, but then flattens out as the active-region magnetism disperses. The streamer belt connects the activeregion domes as illustrated in Figure 8, left panel. The solar wind forms above these magnetic domes or streamer belt; impelled by the large-scale sources of energy and momentum for the solar wind, the interplanetary field then develops its ballerina-skirt structure at higher altitudes.

The warp in the heliospheric current sheet has a natural explanation in terms of the intermittent dominance of activity in the $\mathrm{N}$ or $\mathrm{S}$; roughly we 

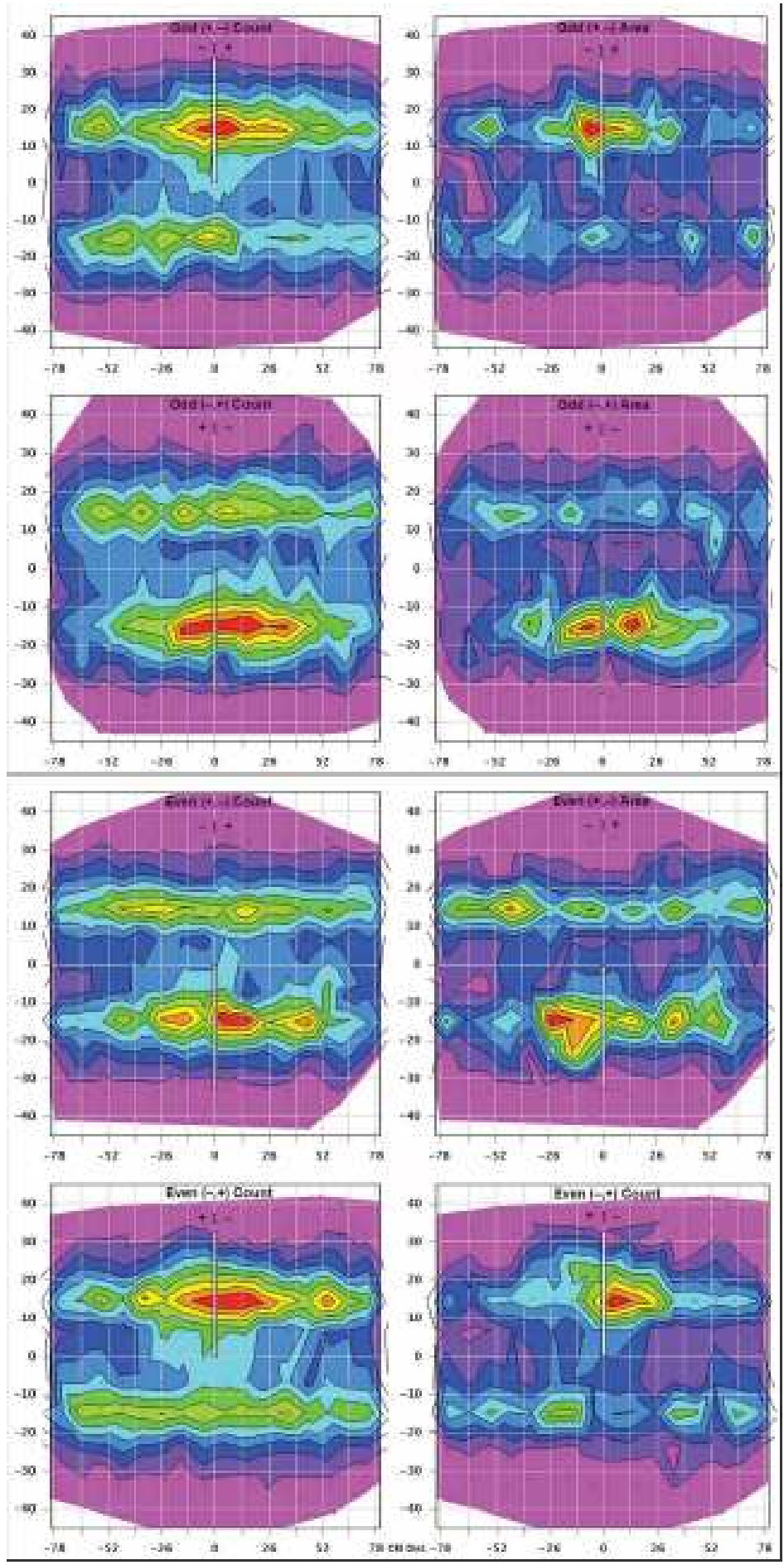

Fig. 10 Summed-epoch analysis for sunspot Group data, based on the Web archive maintained by David Hathaway, comprising some 600 sector boundaries identified for nine solar cycles (16 through 24). The eight panels each show synoptic maps of relative longitude and latitude; the left column is for Group sunspot counts, and the right for areas. The top four show cycles identified as "odd", and the bottom for the "even" ones, and in each case the Hale sector boundary is marked by a vertical rectangle. 
could imagine that bigger magnetic domes or a more inflated streamer zone would simply push the heliospheric current sheet into the opposite hemisphere, considering only the structure resulting from magnetic pressure. This however would put the active regions, sunspots, and flares in between the sector boundaries, not at their very location. We do not have a ready explanation for this but suggest that it may have to do with the inherent latency of large-scale structure development in the corona. Yeates (2014) finds better fits to models with historical knowledge of the field development, rather than an instantaneous vacuum-field model. In this case, the match between activity and sector boundary would be a coincidence, and a manifestation of the time taken for global coronal relaxation.

\section{Conclusions}

The interplanetary magnetic sector structure offers opportunities and puzzles, as we have described. One of the great opportunities lies in its proxy record, which when unfolded will give us a record of the largest-scale solar magnetic fields extending to at least Carrington cycle 9 (from about 1845). The proxy extensions basically confirm that the Hale pattern of polar reversals persists over much longer time scales than the era of direct interplanetary observation, and these records may lead to further discovery. For example, the Hale-boundary segregation extends back in time at least to Cycle 16 already, as described above.

As regards puzzles, we have the basic issue of the connectivity of the solar magnetic field between the photosphere and the heliosphere. This broad subject involves several research communities, specifically those interested in the physical nature of the solar wind. We require intricate $3 \mathrm{D}$ patterns of the sort represented in Figure 7 and these must be dynamical rather than static. Is there a link between the S-web (Antiochos) or interchange models (Fisk) and the Hale sector boundary? The recent discovery of a strong association between the Hale boundaries and the small-scale fields of sunspots and their dynamics (flares) suggests that there might be. The simplification of the photospheric fields as they map into the heliosphere has been found to be inherently dynamic, and so this connection seems like a reasonable one to suggest.

Another open puzzle has to do with the structure of the four-sector pattern. The literature may not convincingly describe the physics behind this persistent corrugation of the heliospheric current sheet (the higher-order wave of the ballerina's skirt).

Acknowledgements We thank the International Space Science Institute for its hospitality during the preparation of this chapter. Author Hudson thanks NASA for support under contract NAS 5-98033 for RHESSI. 


\section{References}

H. Alfvén, On the theory of comet tails. Tellus 9, 92 (1957)

M.D. Altschuler, G. Newkirk, Magnetic Fields and the Structure of the Solar Corona. I: Methods of Calculating Coronal Fields. Solar Phys. 9, 131-149 (1969). doi:10.1007/BF00145734

J.J. Aly, How much energy can be stored in a three-dimensional force-free magnetic field? Astrophys. J. Lett. 375, 61-64 (1991). doi:10.1086/186088

M. Amenomori, Z. Cao, L.K. Ding, Z.Y. Feng, K. Hibino, N. Hotta, Q. Huang, A.X. Huo, H.G. Jia, G.Z. Jiang, S.Q. Jiao, F. Kajino, K. Kasahara, Labaciren, D.M. Mei, L. Meng, X.R. Meng, Mimaciren, K. Mizutani, J. Mu, H. Nanjo, M. Nishizawa, Nusang, A. Oguro, M. Ohnishi, I. Ohta, T. Ouchi, J.R. Ren, T. Saito, M. Sakata, Z.Z. Shi, M. Shibata, T. Shirai, H. Sugimoto, X.X. Sun, K. Taira, Y.H. Tan, N. Tateyama, S. Torii, H. Wang, C.Z. Wen, Y. Yamamoto, X.Y. Yao, G.C. Yu, P. Yuan, T. Yuda, J.G. Zeng, C.S. Zhang, H.M. Zhang, L. Zhang, Zhasang, Zhaxiciren, W.D. Zhou, Direct Evidence of the Interplanetary Magnetic Field Effect on the Cosmic-Ray Shadow by the Sun. Astrophys. J. Lett. 415, 147 (1993). doi:10.1086/187054

M. Amenomori, X.J. Bi, D. Chen, T.L. Chen, W..Y. Chen, S.W. Cui, Danzengluobu, L.K. Ding, C.F. Feng, Z.h. Feng, Z.Y. Feng, Q.B. Gou, Y.Q. Guo, K. Hakamada, H..H. He, Z.T. He, K. Hibino, N. Hotta, H. Hu, J. Hu H. B. an d Huang, H.Y. Jia, L. Jiang, F. Kajino, K. Kasahara, Y. Katayose, C. Kato, K. Kawata, Labaciren, G.M. Le, A.F. Li, H.J. Li, W.J. Li, C. Liu, J.S. Liu, M.Y. Liu, H. Lu, X.R. Meng, K. Mizutani, K. Munakata, H. Nanjo, M. Nishizawa, M. Ohnishi, I. Ohta, H. Onuma, S. Ozawa, X.L. Qian, X.B. Qu, T. Saito, T.Y. Saito, M. Sakata, T.K. Sako, J. Shao, M. Shibata, A. Shiomi, T. Shirai, H. Sugimoto, M. Takita, Y.H. Tan, N. Tateyama, S. Torii, H. Tsuchiya, H. Udo S. an d Wang, H.R. Wu, L. Xue, Y. Yamamoto, Z. Yang, S. Yasue, A.F. Yuan, T. Yuda, L.M. Zhai, H.M. Zhang, J.L. Zhang, X.Y. Zhang, Y. Zhang, Y. Zhang, Y. Zhang, X.X. Zhaxisangzhu an d Zhou, Probe of the solar magnetic field using the "cosmic-ray shadow" of the sun. Phys. Rev. Lett. 111, 011101 (2013). doi:10.1103/PhysRevLett.111.011101. http://link.aps.org/doi/10.1103/PhysRevLett.111.011101

S.K. Antiochos, C.R. DeVore, J.T. Karpen, Z. Mikić, Structure and Dynamics of the Sun's Open Magnetic Field. Astrophys. J. 671, 936-946 (2007). doi:10.1086/522489

S.K. Antiochos, Z. Mikić, V.S. Titov, R. Lionello, J.A. Linker, A Model for the Sources of the Slow Solar Wind. Astrophys. J. 731, 112 (2011). doi:10.1088/0004-637X/731/2/112

S.K. Antiochos, J.A. Linker, R. Lionello, Z. Mikić, V. Titov, T.H. Zurbuchen, The Structure and Dynamics of the Corona-Heliosphere Connection. Space Science Revs. 172, 169-185 (2012). doi:10.1007/s11214-011-9795-7

E. Antonucci, L. Svalgaard, Green Corona and Solar Sector Structure. Solar Phys. 36, 115120 (1974). doi:10.1007/BF00151551

M.J. Aschwanden, Nonlinear Force-free Magnetic Field Fitting to Coronal Loops with and without Stereoscopy. Astrophys. J. 763, 115 (2013). doi:10.1088/0004-637X/763/2/115

M.J. Aschwanden, N.V. Nitta, J.-P. Wuelser, J.R. Lemen, First 3D Reconstructions of Coronal Loops with the STEREO A+B Spacecraft. II. Electron Density and Temperature Measurements. Astrophys. J. 680, 1477-1495 (2008a). doi:10.1086/588014

M.J. Aschwanden, J.-P. Wülser, N.V. Nitta, J.R. Lemen, First Three-Dimensional Reconstructions of Coronal Loops with the STEREO A and B Spacecraft. I. Geometry. Astrophys. J. 679, 827-842 (2008b). doi:10.1086/529542

H.W. Babcock, The Topology of the Sun's Magnetic Field and the 22-YEAR Cycle. Astrophys. J. 133, 572 (1961). doi:10.1086/147060

W.G. Baker, D.F. Martyn, Electric Currents in the Ionosphere. I. The Conductivity. Royal Society of London Philosophical Transactions Series A 246, 281-294 (1953). doi:10.1098/rsta.1953.0016

A. Balogh, G. Erdős, The Heliospheric Magnetic Field. Space Science Revs. 176, 177-215 (2013). doi:10.1007/s11214-011-9835-3

B. Bell, A Long-Term North-South Asymmetry in the Location of Solar Sources of Great Geomagnetic Storms. Smithsonian Contributions to Astrophysics 5, 187 (1962)

L. Biermann, Solar corpuscular radiation and the interplanetary gas. The Observatory $\mathbf{7 7}$, 
109-110 (1957)

J.M. Borrero, K. Ichimoto, Magnetic Structure of Sunspots. Living Reviews in Solar Physics 8, 4 (2011). doi:10.12942/lrsp-2011-4

J.W. Brosius, S.M. White, Radio Measurements of the Height of Strong Coronal Magnetic Fields Above Sunspots at the Solar Limb. Astrophys. J. Lett. 641, 69-72 (2006). doi:10.1086/503774

G.W. Clark, Arrival directions of cosmic-ray air showers from the northern sky. Phys. Rev. 108, 450-457 (1957). doi:10.1103/PhysRev.108.450. http://link.aps.org/doi/10.1103/PhysRev.108.450

W.A. Coles, B.J. Rickett, IPS observations of the solar wind speed out of the ecliptic. Journ. Geophys. Res. 81, 4797-4799 (1976). doi:10.1029/JA081i025p04797

M.L. De Rosa, C.J. Schrijver, G. Barnes, K.D. Leka, B.W. Lites, M.J. Aschwanden, T. Amari, A. Canou, J.M. McTiernan, S. Régnier, J.K. Thalmann, G. Valori, M.S. Wheatland, T. Wiegelmann, M.C.M. Cheung, P.A. Conlon, M. Fuhrmann, B. Inhester, T. Tadesse, A Critical Assessment of Nonlinear Force-Free Field Modeling of the Solar Corona for Active Region 10953. Astrophys. J. 696, 1780-1791 (2009). doi:10.1088/0004$637 \mathrm{X} / 696 / 2 / 1780$

L.A. Fisk, N.A. Schwadron, The Behavior of the Open Magnetic Field of the Sun. Astrophys. J. 560, 425-438 (2001). doi:10.1086/322503

L.A. Fisk, L. Zhao, The heliospheric magnetic field and the solar wind during the solar cycle, in IAU Symposium, ed. by N. Gopalswamy, D.F. Webb IAU Symposium, vol. 257, 2009, pp. 109-120. doi:10.1017/S1743921309029160

R.A. Frazin, P. Lamy, A. Llebaria, A.M. Vásquez, Three-Dimensional Electron Density from Tomographic Analysis of LASCO-C2 Images of the K-Corona Total Brightness. Solar Phys. 265, 19-30 (2010). doi:10.1007/s11207-010-9557-9

E. Friis-Christensen, K. Lassen, J.M. Wilcox, W. Gonzalez, D.S. Colburn, Interplanetary Magnetic Sector Polarity from Polar Geomagnetic Field Observations. Nature Physical Science 233, 48-50 (1971). doi:10.1038/physci233048a0

A. Gulbrandsen, On the variation of the coronal $\lambda 5303$ intensity relative to the interplanetary and solar magnetic sector structure, and to geomagnetic activity. Planetary \& Space Sci. 21, 703-707 (1973). doi:10.1016/0032-0633(73)90086-X

J.T. Hoeksema, J.M. Wilcox, P.H. Scherrer, The structure of the heliospheric current sheet 1978-1982. Journ. Geophys. Res. 88, 9910-9918 (1983). doi:10.1029/JA088iA12p09910

R.B. Leighton, Transport of Magnetic Fields on the Sun. Astrophys. J. 140, 1547 (1964). doi:10.1086/148058

R.B. Leighton, A Magneto-Kinematic Model of the Solar Cycle. Astrophys. J. 156, 1 (1969). doi:10.1086/149943

I.M. Livshits, V.N. Obridko, Variations of the dipole magnetic moment of the sun during the solar activity cycle. Astronomy Reports 50, 926-935 (2006). doi:10.1134/S1063772906110060

M. Lockwood, Reconstruction and Prediction of Variations in the Open Solar Magnetic Flux and Interplanetary Conditions. Living Reviews in Solar Physics 10, 4 (2013). doi:10.12942/lrsp-2013-4

M. Lockwood, M. Owens, A.P. Rouillard, Excess open solar magnetic flux from satellite data: 2. A survey of kinematic effects. Journal of Geophysical Research (Space Physics) 114, 11104 (2009). doi:10.1029/2009JA014450

D. Mackay, A. Yeates, The Sun's Global Photospheric and Coronal Magnetic Fields: Observations and Models. Living Reviews in Solar Physics 9, 6 (2012). doi:10.12942/lrsp2012-6

S.M. Mansurov, New Evidence of a Relationship between Magnetic Fields in Space and on Earth. Geomagnetism and Aeronomy 9, 622 (1970)

K. Mursula, T. Hiltula, Bashful ballerina: Southward shifted heliospheric current sheet. Geophys. Res. Lett. 30, 2135 (2003). doi:10.1029/2003GL018201

H. Nevanlinna, Gauss's H-Variometer at the Helsinki Magnetic Observatory 18441912. Journal of geomagnetism and geoelectricity 49(10), 1209-1215 (1997). doi:10.5636/jgg.49.1209

M.J. Owens, R.J. Forsyth, The Heliospheric Magnetic Field. Living Reviews in Solar Physics 10, 5 (2013). doi:10.12942/lrsp-2013-5 
M.J. Owens, N.U. Crooker, M. Lockwood, How is open solar magnetic flux lost over the solar cycle? Journal of Geophysical Research (Space Physics) 116, 4111 (2011). doi:10.1029/2010JA016039

M.J. Owens, C.N. Arge, N.U. Crooker, N.A. Schwadron, T.S. Horbury, Estimating total heliospheric magnetic flux from single-point in situ measurements. Journal of Geophysical Research (Space Physics) 113, 12103 (2008). doi:10.1029/2008JA013677

E.N. Parker, Dynamics of the Interplanetary Gas and Magnetic Fields. Astrophys. J. 128, 664 (1958). doi:10.1086/146579

E.R. Priest, P. Démoulin, Three-dimensional magnetic reconnection without null points. 1. Basic theory of magnetic flipping. Journ. Geophys. Res. 100, 23443-23464 (1995). doi:10.1029/95JA02740

R.L. Rosenberg, P.J. Coleman Jr., Heliographic latitude dependence of the dominant polarity of the interplanetary magnetic field. Journ. Geophys. Res. 74, 5611 (1969). doi:10.1029/JA074i024p05611

K.H. Schatten, J.M. Wilcox, N.F. Ness, A model of interplanetary and coronal magnetic fields. Solar Phys. 6, 442-455 (1969). doi:10.1007/BF00146478

M. Schulz, Interplanetary sector structure and the heliomagnetic equator. Astrophys. \& Space Sci. 24, 371-383 (1973). doi:10.1007/BF02637162

J.A. Simpson, M. Zhang, S. Bame, A Solar Polar North-South Asymmetry for Cosmic-Ray Propagation in the Heliosphere: The ULYSSES Pole-to-Pole Rapid Transit. Astrophys. J. Lett. 465, 69 (1996). doi:10.1086/310127

E.J. Smith, A. Balogh, Ulysses observations of the radial magnetic field. Geophys. Res. Lett. 22, 3317-3320 (1995). doi:10.1029/95GL02826

P.A. Sturrock, Maximum energy of semi-infinite magnetic field configurations. Astrophys. J. 380, 655-659 (1991). doi:10.1086/170620

L. Svalgaard, Sector structure of the interplanetary magnetic field and daily variation of the geomagnetic field at high latitudes. Danish Met. Inst. Geophys. Papers R-6 (1968)

L. Svalgaard, Interplanetary magnetic-sector structure, 1926-1971. Journ. Geophys. Res. 77, 4027 (1972). doi:10.1029/JA077i022p04027

L. Svalgaard, Polar cap magnetic variations and their relationship with the interplanetary magnetic sector structure. Journ. Geophys. Res. 78, 2064 (1973). doi:10.1029/JA078i013p02064

L. Svalgaard, J.M. Wilcox, Long-term evolution of solar sector structure. Solar Phys. 41, 461-475 (1975). doi:10.1007/BF00154083

L. Svalgaard, J.M. Wilcox, The Hale solar sector boundary. Solar Phys. 49, 177-185 (1976). doi:10.1007/BF00221492

L. Svalgaard, I.G. Hannah, H.S. Hudson, Flaring Solar Hale Sector Boundaries. Astrophys. J. 733, 49 (2011). doi:10.1088/0004-637X/733/1/49

L. Svalgaard, J.M. Wilcox, T.L. Duvall, A Model Combining the Polar and the Sector Structured Solar Magnetic Fields. Solar Phys. 37, 157-172 (1974). doi:10.1007/BF00157852

L. Svalgaard, J.M. Wilcox, P.H. Scherrer, R. Howard, The sun's magnetic sector structure. Solar Phys. 45, 83-91 (1975). doi:10.1007/BF00152219

G.S. Vaiana, A.S. Krieger, A.F. Timothy, Identification and Analysis of Structures in the Corona from X-Ray Photography. Solar Phys. 32, 81-116 (1973). doi:10.1007/BF00152731

J.E. Vernazza, E.H. Avrett, R. Loeser, Structure of the solar chromosphere. III - Models of the EUV brightness components of the quiet-sun. Astrophys. J. Supp. 45, 635-725 (1981). doi:10.1086/190731

M.V. Vokhmyanin, D.I. Ponyavin, Sector structure of the interplanetary magnetic field in the nineteenth century. Geophys. Res. Lett. 40, 3512-3516 (2013). doi:10.1002/grl.50749

Y.-M. Wang, E. Robbrecht, Asymmetric Sunspot Activity and the Southward Displacement of the Heliospheric Current Sheet. Astrophys. J. 736, 136 (2011). doi:10.1088/0004$637 \mathrm{X} / 736 / 2 / 136$

Y.-M. Wang, N.R. Sheeley Jr., Magnetic flux transport and the sun's dipole moment - New twists to the Babcock-Leighton model. Astrophys. J. 375, 761-770 (1991). doi:10.1086/170240

Y.-M. Wang, N.R. Sheeley Jr., On the Fluctuating Component of the Sun's Large-Scale Magnetic Field. Astrophys. J. 590, 1111-1120 (2003). doi:10.1086/375026 
Y.-M. Wang, J. Lean, N.R. Sheeley, The long-term variation of the sun's open magnetic flux. Geophysical Research Letters 27(4), 505-508 (2000). doi:10.1029/1999GL010744. http://dx.doi.org/10.1029/1999GL010744

J.M. Wilcox, Inferring the interplanetary magnetic field by observing the polar geomagnetic field. Reviews of Geophysics and Space Physics 10, 1003-1014 (1972). doi:10.1029/RG010i004p01003

J.M. Wilcox, R. Howard, A Large-Scale Pattern in the Solar Magnetic Field. Solar Phys. 5, 564-574 (1968). doi:10.1007/BF00147021

J.M. Wilcox, N.F. Ness, Quasi-Stationary Corotating Structure in the Interplanetary Medium. Journ. Geophys. Res. 70, 5793-5805 (1965). doi:10.1029/JZ070i023p05793

A.R. Yeates, Coronal Magnetic Field Evolution from 1996 to 2012: Continuous Non-potential Simulations. Solar Phys. 289, 631-648 (2014). doi:10.1007/s11207-013-0301-0

X. Zhao, J.T. Hoeksema, Predicting the heliospheric magnetic field using the current sheetsource surface model. Advances in Space Research 16, 181 (1995). doi:10.1016/02731177(95)00331-8

T.H. Zurbuchen, A new view of the coupling of the sun and the heliosphere. Annual Review of Astronomy and Astrophysics 45(1), 297-338 (2007). doi:10.1146/annurev.astro.45.010807.154030 